\title{
Construire une culture commune entre enseignants spécialisés et infirmiers en hôpital de jour : vers une identification d'objets migrants
}

\author{
Valérie GUINARD* \\ Enseignante spécialisée \\ Formatrice à l'inspection académique de Loire-Atlantique
}

\author{
Marie TOULLEC-THÉRY** \\ Maître de conférences, CREN \\ Université de Nantes et Espé
}

Résumé :

\begin{abstract}
Les enseignants spécialisés, en poste dans des hôpitaux de jour, ont une place légitimée avec la création d'unité d'enseignement (2009). Leur rôle et leur mission sont malgré tout encore questionnés et les mises en æuvre ne vont pas de soi.

Quels liens peuvent-ils en effet tisser avec des professionnels de cultures différentes voire concurrentes? Il importe alors de revenir sur la culture de la séparation et de la différenciation telle qu'elle existait dans ces univers de soin et de repérer des alliances entre soin et enseignement-apprentissage, entre des articulations des pratiques à visée thérapeutique, pédagogique et éducative. En venant bousculer les habitudes d'action avec l'élaboration de temps de rencontre co-construits par les professionnels et le chercheur dans une ingénierie coopérative, cette recherche fait coopérer des acteurs pluricatégoriels pour envisager, dans une transformation " gagnant-gagnant», de nouvelles pratiques professionnelles.
\end{abstract}

Mots-clés : hôpital de jour, enseignement spécialisé, besoin éducatif particulier, coopération, territoires professionnels, équipe pluricatégorielle.

Building a common culture between specialized teachers and day hospitals: towards an identification of migrant objects

Summary:

Specialized teachers working within a day hospital setting, have been credited a legitimate position since the implementation of teaching units (2009). However, their role and mission are still being questionned and these implementations are not obvious.

What connections can they indeed create with professionals working in different, if not rival, cultures? As a result, it is important to review the culture of separation and differentiation as it existed within these treatment services and to identify the alliances between treatment and pedagogy, as well as the hinges between therapeutic, teaching and educational goals.

As the implementation of meeting sessions co-designed by both professionals and scientists in a cooperative engineering manner disrupts the usual steps taken, this research generates the cooperation of multi-category actors to consider, in a win-win situation, new professional practices.

Keywords: day hospital, specialized teaching, inclusive, specific education needs, cooperation, professional territories, multi-category team

\footnotetext{
*valerie.guinard@ac-nantes.fr

** marie.toullec-thery@univ-nantes.fr
} 
Les services de pédopsychiatrie, rattachés à un Centre Hospitalier, sont organisés, dans les années 70, en secteur. Ils offrent, à proximité des patients, soit un espace de consultation, soit une prise en charge totale, avec les hôpitaux de jour. Un premier changement, introduit par la loi de 1975, pose une «obligation éducative ». Des enseignants spécialisés entrent alors dans cette institution. Cette mesure répond à "la nécessité de faire acquérir des apprentissages scolaires de base 》 (Gossot, 2007) à ces enfants-patients, nécessité portée par des médecins et des parents. La loi de 2005 opère une nouvelle modification : tout enfant est prioritairement scolarisé dans son école de quartier. D'une prise en charge à plein temps, les enfants se voient désormais proposer des temps partagés entre institution de soin et école ordinaire. L'arrêté sur la création des Unités d'Enseignement ${ }^{1}$ (2009) préconise ainsi des imbrications entre institution de soin, enseignement spécialisé et classe ordinaire de référence de l'enfant. Les professions du soin, tout comme celles de l'enseignement, sont donc aux prises avec ce mouvement.

Le contexte de notre étude se situe dans un service de pédopsychiatrie, et plus précisément dans une unité de l' hôpital de jour qui accueille des enfants de 6 à 12 ans, à temps partiel. Sous la responsabilité d'un médecin pédopsychiatre, cette unité de soin et d'enseignement spécialisé (USP) a pour objectif spécifique de remettre l'enfant dans une dynamique d'apprentissage. Elle est composée d'une équipe restreinte de 3 infirmiers, 2 enseignants et un éducateur spécialisé. Les enfants fréquentent obligatoirement les deux systèmes (soin et enseignement-apprentissage), aux tutelles différentes (Sanitaire et Éducation nationale). Le système de soin est implanté dans le service de pédopsychiatrie et l'Unité d'enseignement (UE) se situe, quant à elle, au sein d'une école publique (avec deux espaces classes).

Les enfants adressés à l'USP présentent tous des troubles avérés des apprentissages qui perturbent leur inscription en tant qu'élève dans leur école de secteur, sans pour autant que ces troubles n'entrainent une reconnaissance de handicap. Leur admission nécessite une prescription médicale, effectuée par un médecin de consultation et l'accord des parents. Après validation du dossier par le médecin responsable de l'USP, un temps d'observation de l'enfant, au sein de l'Unité et un échange avec l'enseignant de l'école sont mis en place afin de proposer un projet personnalisé, cohérent, de prise en charge.

Nous questionnons, dans cet article, la manière dont les enseignants spécialisés et deux des infirmiers opèrent pour construire une cohérence dans le parcours de l'enfant, entre le système de soin et le système d'enseignement.

Dans le cadre de la recherche, ces professionnels se sont engagés dans un double dispositif qui consiste en l'organisation et la mise en œuvre :

1. d'une "médiation cuisine», qui vise le croisement du thérapeutique, de l'éducatif, du «pédagogique et didactique » (Emery et Pelgrims, 2015), tel que fixé dans les enjeux de l'Unité. Cette «médiation cuisine» fait travailler, dans le système d'enseignementapprentissage, un texte de recette et, dans le système de soin, aboutit à la réalisation de cette recette. Chaque système identifie des enjeux, dans le champ des apprentissages et dans celui du thérapeutique,

\footnotetext{
${ }^{1}$ Arrêté du 2 avril 2009 précisant les modalités de création et d'organisation d'unités d'enseignement dans les établissements et services médico-sociaux ou de santé.
} 
2. d'une ingénierie coopérative (Sensevy, 2011), où chercheurs et professionnels se sont réunis six fois ${ }^{2}$ pour analyser leurs pratiques et en concevoir de nouvelles. Cette ingénierie coopérative fonctionne comme un collectif chercheur-professionnels « en transaction, pour la production de nouvelles capacités individuelles et collectives » (Ibid, p. 681).

Il s'agit alors, dans cette recherche, de comprendre comment des enseignants et des infirmiers via une médiation «cuisine», articulent des temps thérapeutiques et des temps d'enseignementapprentissages, pour que les enfants de l'Unité développent concomitamment des savoirs, des savoirfaire et des savoir-être. Parallèlement, nous questionnons la portée de l'ingénierie coopérative pour déceler si elle amorce une culture commune et modifie alors les pratiques professionnelles.

L'analyse que nous avons menée, inscrite dans une dimension didactique, se centre, d'une part, sur les interactions langagières professionnels-enfants, lors des séances dans le système de soin et dans le système d'enseignement-apprentissage. Des mots, des allusions, des références à l'autre système pourraient, dans ces interactions, attester de signes d'une constitution de liens (ou non) entre les deux espaces. Nous traquons ainsi le degré d'articulation ou d'étanchéité entre les deux systèmes que sont le soin et l'enseignement.

D'autre part, nous analysons ce qui se passe au sein des réunions d'ingénierie et décelons ainsi ce qui fait que les pratiques «bougent», les traces (dans les discours) qui attesteraient de la construction d'une culture commune et partagée (Emery, 2011).

Notre propos, dans cet article, s'attachera plus particulièrement aux infirmiers.

\section{LE CADRE INSTITUTIONNEL : D'INSTITUTIONS AUX FRONTIERES MARQUEES VERS UN DESIR DE COOPERATION}

La scolarisation des élèves « troublés » est ancienne et n'est pas évidente à retracer (Ruchat, 2003). Exposer le poids de la construction historique permet pourtant de comprendre ce qui mène aux manières de faire et de penser aujourd'hui l'hôpital de jour.

Si la loi du 15 avril 1909, avec la création des Classes de Perfectionnement, ouvre l'école aux « arriérés », peu d'entre eux y seront scolarisés. Leur prise en charge incombe en effet surtout aux services hospitaliers, avec une distinction de niveaux d'éducabilité, via une classification entre les « anormaux d'hospice » et les « anormaux pédagogiques », selon les termes d'Alfred Binet. L'histoire de l'éducation spéciale commence ici, cette dénomination disparaitra seulement avec la loi du 11 février 2005.

Les années soixante voient la prise en charge des enfants inadaptés comme une pratique fondée sur la défectologie, où la nosographie domine. Cette période correspond à celle de la ségrégation où les lieux de soin sont lieu de réparation. L'idée principale est de «sortir» l'enfant de son environnement ordinaire et de le placer dans une institution externalisée où des réponses adaptées le guériront ou l'amélioreront. En d'autres termes, c'est une construction filiarisée qui exclut selon une classification de la différence, perçue comme déficience.

\footnotetext{
${ }^{2}$ Ces temps étaient déjà inscrits à l'emploi du temps des professionnels depuis un an, mais jusque lors très peu investis.
} 
Les années soixante-dix marquent l'apparition des services de pédopsychiatrie et des hôpitaux de jour pour enfants. Certains emploient progressivement des enseignants et promeuvent ainsi «la légitimité d'un enseignement dans les établissements spécialisés » (Dorison, 2015). L'apprentissage scolaire se pare alors d'une «valeur structurante, il contribue à organiser la psyché selon des lignes de force » (Hochmann, 2013). Si la loi de 1975 pose une « obligation éducative » pour les enfants handicapés et met ainsi fin aux «niveaux d'éducabilité », elle ne règle pas pour autant concrètement la question de la scolarité de ces élèves, ni des conditions de l'articulation entre les différentes institutions (Chauvière et Plaisance, 2008). Leurs constructions historiques sont en effet toujours fondées sur la séparation, avec ce que Chauvière et Plaisance (ibid.) nomment des " concurrences territoriales 》.

Plus récemment, le décret n²009-378 du 2 avril 2009 s'oppose à la pensée d'un monde dichotomique. Il promeut la coopération entre les différents acteurs de l'accompagnement et de la scolarisation des enfants à besoins éducatifs particuliers. Ses mesures d'application corroborent cette volonté de faire de la scolarisation un but commun à tous les acteurs. L'hôpital de jour ne se centre alors plus uniquement sur « l'enfant, objet de soins », mais participe à la continuité de son parcours de formation, " c'est-à-dire de préparer et compléter l'enseignement reçu en milieu ordinaire » (Benoit, 2012), et notamment de rendre les savoirs accessibles à l'élève. La création des unités d'enseignement, engagée au même moment par l'arrêté ${ }^{3}$ de 2009 , constitue alors une étape fondamentale. La coopération entre enseignants spécialisés et professionnels des établissements médico-sociaux ou de santé s'y avère un véritable enjeu parce qu'il y a nécessité d'un projet transdisciplinaire. Duquesne-Belfais (2008) défend que «doivent alors être identifiés les facteurs environnementaux qui peuvent, soit faire obstacle, soit faciliter le développement des capacités pour agir en fonction d'eux». D'une logique professionnelle de "cohabitation », on arrive à celle de coopération où "la scolarisation des élèves présentant un besoin éducatif particulier est un acte de co-production mobilisant une pluralité d'acteurs appartenant à des univers organisationnels distincts " (Ebersold, 2012). Il s'agit alors aussi d'une articulation des savoirs entre le monde du soin et celui de l'enseignement, tant pour les professionnels que pour les des enfants. Mais comment atteindre cet objectif ambitieux?

\section{ÉLEMENTS DU CADRE THEORIQUE}

Dans le cadre de notre étude, nous empruntons à Tambone (2014) et à la théorie des systèmes de Chevallard (1995), leur approche systémique, pour comprendre ce qui se passe lors des pratiques effectives dans les deux systèmes que sont l'enseignement et le soin. Nous envisageons alors les systèmes de soin et d'enseignement-apprentissage, dans une dimension anthropologique, comme deux systèmes où enseignants spécialisés et infirmiers ont l'intention de modifier « quelque chose » chez les enfants pour accroitre leur "puissance d'agir ${ }^{4}$ (Sensevy et Mercier, 2007 ; Sensevy, 2011). Il s'agit donc, à ce titre, de deux systèmes que nous considérons comme didactiques, même si ce « quelque chose » à modifier, n'est pas de la même nature dans les deux systèmes. Dans l'USP, nous considérons que le système de soin est le système central et le système d'enseignement-apprentissage, le système auxiliaire (Tambone, 2014). En effet, Emery (2017) avance que, dans les contextes

\footnotetext{
${ }^{3}$ En application des articles D. 351-17 à D. 351-20 du code de l'éducation

${ }^{4}$ Ce qui est développé dans chaque système engage l'élève à développer des stratégies et des savoirs de divers ordres, que nous considérons, à la suite de Sensevy (2011) comme une " puissance d'agir ».
} 
d'institutions spécialisées, « l'enseignement n'est qu'un système participant au mandat thérapeutique de l'institution ». L'enjeu de l'USP est alors d'articuler les deux systèmes pour que les enfants ne les considèrent pas étanches, et consolident ainsi des savoirs ${ }^{5}$.

- La mise en articulation des deux systèmes, est anticipée dans l'ingénierie coopérative. Il s'agit alors d'identifier ce qui peut devenir un « objet migrant », qui "passe d'un système à l'autre par l'intermédiaire de l'élève » (Tambone, Ibid.). C'est en effet «l'objet migrant» qui engagerait une percolation entre les deux systèmes. Le travail entre les professionnels, au sein de l'ingénierie devient alors soumis à la construction d'un contexte cognitif commun, condition qui permettrait aux enfants d'être en mesure de décontextualiser des savoirs de leur situation d'apprentissage d'origine et de les recontextualiser dans une autre. Cette circulation d'objets migrants diminuerait alors le "degré d'étanchéité » entre les deux systèmes.

- La consolidation des savoirs, au sein de la «médiation cuisine», est double : il s'agit d'être capable de réaliser une recette, dans le système de soin, en construisant des savoir-faire et des savoir-être ${ }^{6}$, tout en mobilisant des savoirs scolaires effectués dans le système d'enseignement, mettant en jeu des compétences en lecture/écriture et mathématiques (comparer et mesurer des masses) du cycle 2. Ce double système articulé enseignement-soin favoriserait alors le retour de l'enfant dans sa classe ordinaire.

Dans notre recherche, nous avons alors, en identifiant les savoirs en cours, traqué les objets migrants qui transitent entre le système de soin et le système d'enseignement et tenté de déceler en quoi ils engagent les deux systèmes à devenir moins étanches.

Pour analyser les pratiques effectives dans les deux systèmes, nous avons mis au travail le cadre théorique de l'action conjointe en didactique-TACD- (Sensevy 2007, 2011). Dans tout jeu didactique, "B accompagne A dans ce jeu. B gagne lorsque A gagne » (Sensevy, 2007, p.20). Dans le système d'enseignement, les enseignants gagnent quand les élèves ${ }^{7}$ arrivent à lire (ou tout au moins à repérer les mots-clés) et s'emparent des notions de quantité et de mesure. Dans le système de soin, les infirmiers gagnent quand les enfants réalisent ensemble la recette jusqu'à son terme, en ajustant leurs comportements aux autres, à la situation et en acceptant de réinvestir des connaissances qu'ils ont déjà. Les médiations thérapeutiques à caractère éducatif ou pédagogique (Marciano, 2009), ainsi la médiation cuisine, peuvent donc être vues comme des situations qui ont l'intention de modifier les savoirs de l'enfant, dans leurs différentes dimensions, et de favoriser ainsi son développement social et sa construction identitaire. Si l'enseignant spécialisé organise le jeu et accompagne l'apprenant pour que celui-ci puisse gagner au jeu, il en est de même entre l'infirmier et le patient. On peut donc parler, de manière très anthropologique, de jeu d'apprentissage dans le système d'enseignement, mais également lors «d'autres situations de travail» (Sensevy, 2002). Nous utiliserons donc un même quadruplet de descripteurs pour analyser les systèmes d'enseignement et de soin. Au cours de son action, l'enseignant spécialisé ou l'infirmier va en effet définir le jeu, le dévoluer, le réguler puis l'institutionnaliser $^{8}$. Nous nous intéresserons plus particulièrement à la dévolution, qui se définit

\footnotetext{
${ }^{5}$ Un savoir étant considéré dans les diverses dimensions du triptyque, savoir, savoir-faire et savoir être.

${ }^{6}$ Mener une action à son terme, trouver sa place au sein d'un groupe, entretenir une relation à l'autre avec une bonne distance, coopérer, accepter des règles, reprendre confiance en soi, devenir plus autonome, ...

${ }^{7}$ Le groupe constitué par l'équipe multiprofessionnelle est de quatre enfants, tous scolarisés en cycle 2 . La « médiation cuisine » a été proposée en réponse à leurs besoins identifiés.

${ }^{8}$ Il s'agit ainsi, dans un premier temps, de transmettre les règles « constitutives, définitoires du jeu » (Sensevy, 2007, p.28) c'est-à-dire de « définir le jeu ». Puis pour que les enfants acceptent de jouer le jeu, « de jouer au bon jeu » (Sensevy,
} 
comme la part de responsabilité de la situation que l'adulte « sachant» laisse à l'enfant « qui ne sait pas encore ». Nous nous fonderons aussi sur le couple réticence-expression. Comme le travail engagé dans l'ingénierie didactique a pour ambition d'articuler les systèmes d'enseignement et de soin, chaque professionnel en faisant référence à l'autre système peut alors ne pas tout dire (réticence) et privilégier la recontextualisation par les élèves de savoirs effectués dans l'autre système.

Dans notre recherche, nous analysons donc ce que produit l'articulation entre le travail d'ingénierie et les pratiques effectives. Nous tentons de déceler si cette manière de travailler module les responsabilités respectives des professionnels et des élèves en situation.

\section{ÉLEMENTS DU CADRE METHODOLOGIQUE}

Le terrain de recherche est construit sur une tentative de résolution de problème, issu des pratiques au quotidien, qui fait signe à une équipe de professionnels. C'est donc une recherche « avec » plutôt que « sur » les praticiens (Lieberman, 1986) où l' «un des enjeux principaux du travail en équipe est la transformation des représentations individuelles vers des représentations communes et partagées » (Emery, 2011). La particularité de cette recherche est de mettre en place un lieu de partage de pratiques, fondé sur un principe de symétrie (Sensevy, 2011). Le collectif (2 enseignants, 3 infirmiers, un éducateur, un chercheur) enquête pour résoudre ce problème et est amené à imaginer ensemble une ou plusieurs solutions prenant une forme inédite (Perraud, 2018). Le chercheur contribue, comme les autres, à l'ingénierie coopérative (Sensevy, 2011 ; Perraud, 2018). Pour sa part, il a initié la lecture d'articles centrés sur le langage commun au sein d'équipes pluricatégorielles pour susciter des questions, pour engager, avec cet arrière-fond commun, une « réflexivité conjointe » (Desgagné et al., 2001) et ainsi construire les conditions qui permettraient aux enfants de gagner de la «puissance d'agir » dans la « médiation cuisine ». Dans cette ingénierie coopérative la place du chercheur n'est pas transparente, il assume une certaine dissymétrie car le chercheur est celui qui « tient la lanterne » (Marlot, Toullec-Théry et Daguzon, 2017).

Notre recueil de données repose sur :

- quatre enregistrements vidéo, deux réalisés lors de séances d'enseignement-apprentissages avec les enseignants et deux, lors des séances thérapeutiques avec les infirmiers. Les deux premières séances (T0), respectivement en classe spécialisée et dans l'espace soin, ont été filmées au tout début du travail coopératif. Elles représentent donc un état initial des pratiques professionnelles (enseignants spécialisés et infirmiers) lors de la «médiation cuisine ». Les secondes ont été filmées au bout de 5 mois ;

- des enregistrements audio faits lors des six réunions de l'ingénierie coopérative.

Quilio, 2002), le professeur va devoir pour cela le « réguler» tout en, « dévoluant le jeu » pour rendre l'élève responsable de ses apprentissages. Enfin l'institutionnalisation consiste à « ce que le professeur fait pour que tel ou tel comportement, telle ou telle assertion, ou telle ou telle connaissance, soient considérés comme légitimes, vrais et attendus, dans l'institution » (Sensevy, 2001).

${ }^{9}$ « Celui qui sait quelque chose partage ce quelque chose avec les autres afin que le collectif travaille ensemble à la construction de solutions adéquates à la logique d'une pratique » (Perraud, 2019). 
Chaque catégorie de professionnels a isolé deux épisodes significatifs de ses pratiques, issus de la première séance. Ils les ont présentés au collectif. En visionnant les vidéos, les professionnels ont, avec une certaine surprise, découvert, leur propre pratique professionnelle, mais aussi les pratiques des autres professionnels. Les enseignants ont ainsi présenté un épisode où il s'agissait d'identifier, dans le texte de la recette, le titre, la liste des ingrédients et les étapes de la préparation, ce qui leur permettait ensuite de faire élaborer des hypothèses de lecture et de produire une fiche recette personnalisée, selon les besoins en lecture de chaque élève. Or cette fiche n'est pas utilisée par les infirmiers qui lui préfèrent la version (sans adaptation) du livre de recette. Est donc apparue en acte la rupture entre les deux systèmes de soin et d'enseignement. Le chercheur a alors donné un statut à cette rupture, perçue par les professionnels lors du visionnage, et fait le lien avec la notion d'objets migrants. Le collectif a alors amorcé un travail où il s'agissait de déceler quels étaient les savoirs attendus et ceux qui étaient réellement travaillés dans chaque système, mais aussi les manières dont ces savoirs circulaient entre les systèmes de soin et d'enseignement.

Nous avons réitéré ce même dispositif de recueil de données après cinq mois de travail de l'ingénierie coopérative et sélectionné (par le chercheur cette fois-ci) de nouveaux épisodes, issus des deux nouvelles séances filmées (T1). Nous y avons porté une double attention : Chacun des systèmes rappelle-t-il à la mémoire des élèves ce qui s'est fait dans l'autre système ? Comment chaque professionnel laisse-t-il aux enfants l'opportunité de prendre la responsabilité de la situation?

Dans le souci d'évaluer les impacts du dispositif d'ingénierie sur les pratiques effectives, le chercheur a repris l'ensemble des données issues :

1) des réunions d'ingénierie, à partir d'écoutes multiples et attentives, pour repérer les expressions qui attestent d'une construction par les professionnels d'un contexte cognitif commun, c'est-à-dire, d'un entre-deux qui soit un espace partagé de langage et de pensée ;

2) des épisodes choisis en $\mathrm{T} 1$, entièrement retranscrits, pour sélectionner, sous le prisme du quadruplet de descripteurs (Sensevy, 2007), les énoncés in situ où enseignants et infirmiers définissent la situation, dévoluent la responsabilité de la situation aux enfants et régulent l'activité des élèves ou des enfants.

\section{QUELQUES RESULTATS}

Des réunions d'ingénierie, clé de voute à la connaissance réciproque des attentes et des intentions de chaque profession

L'analyse de la première réunion d'ingénierie coopérative, au début de la recherche, montre une résistance initiale des acteurs du système de soin à toute coopération. Le discours des infirmiers souligne en effet la volonté de conserver enseignement et soin en deux univers cloisonnés, voire étanches et de ne "pas lâcher trop de choses de l'ordre de la psy comme on est tenu par le secret professionnel ». Les infirmiers trouvent de surcroit que c'est aux enseignants «de faire un effort d'acculturation ». Les enseignants spécialisés comme les infirmiers s'accordent aussi, à ce stade de l'ingénierie, à trouver que tout oppose ces deux corps de métier : ils n'y décèlent pas, d'emblée, des analogies dans leurs actions.

L'analyse de l'ensemble des réunions d'ingénierie, mises en œuvre sur cinq mois, montre qu'elles ont favorisé un climat propice à bousculer des allants de soi. Ainsi, les visionnages d'épisodes sélectionnés 
ont été cruciaux parce qu'ils ont très vite (dès T0) amené les professionnels vers la mise au jour de décalages entre ce qu'ils font et ce qu'ils ont chacun le sentiment de faire. Ainsi un des infirmiers dit : "dans mon ressenti, j'avais l'impression que ... mais en fait non », "j’ai été agréablement surpris par la façon dont ... ». Cette déstabilisation occasionnée par le visionnage des épisodes les met à distance de leurs propres pratiques et leur permet de découvrir la pratique des autres, d'en déconstruire des représentations et de s'engager alors à analyser de manière critique leurs manières de faire entre système de soin et système d'enseignement. Ces réunions d'ingénierie contribuent donc à la transformation du rapport infirmier/enseignant.

\section{L'ingénierie coopérative comme lieu de repérage d'événements, déclencheurs d'une articulation entre les territoires et des pratiques}

Pour rendre nécessaire tout changement ou modulation de pratiques, une perturbation, un problème, un dilemme doivent venir déstabiliser des habitudes (Altet, 2000). C'est ce qui se passe dans notre étude.

Une des déstabilisations advient de manière involontaire. Elle résulte d'un malentendu entre les deux systèmes. Suite à une modification du déroulé d'une activité dans le système d'enseignement, les élèves ont seulement étudié le texte d'une recette, sans produire de fiche recette. Les soignants n'en ont pas été prévenus et ne peuvent donc l'utiliser comme support d'aide à la réalisation de la recette, comme il en est désormais usage (les infirmiers ont en effet cessé de prendre appui sur la recette du livre de cuisine). Un des enfants se montre alors en mesure de se remémorer la recette découverte dans le système classe. "Il avait [en tête] toute la recette du pain qu'il a pu nous dire », précise ainsi un des infirmiers lors de la réunion d'ingénierie suivante. Cet enfant utilise donc spontanément le travail effectué dans le système d'enseignement dans le système de soin : c'est la première fois qu'un lien explicite est effectué entre les deux systèmes. Cet incident montre aux professionnels la possibilité dont s'est saisi un enfant, à partir de son expérience dans le système d'enseignement, de réinvestir ses savoirs dans le cadre du soin, tout en permettant aux soignants de garantir leurs objectifs de prise en charge. Les professionnels reconnaissent alors que cette rupture dans le déroulement habituel de la médiation n'entraîne pas une perte de territoire, mais, plutôt, un enrichissement. Nait alors une pratique jusque lors inédite. Cette articulation reconnue comme bénéfique devient une pratique à renouveler.

Une seconde modification advient dans les pratiques et dans le répertoire d'actions de chaque catégorie de personnel. Elle fait suite à l'introduction d'un nouvel objet, une balance électrique, qui est reconnu et trouve instantanément une place dans le système d'enseignement et dans le système de soin (ce qui n'avait pas été le cas pour la fiche recette ${ }^{10}$ ). La pesée, apprise en classe, devient un moyen réinvesti dans le système de soin.

\section{Infirmier : Tu te rappelles ... pourquoi on a besoin de ça?}

\footnotetext{
${ }^{10}$ La médiation cuisine existe depuis le début de l'institution, mais avec initialement une seule visée thérapeutique. Une fiche faisait alors déjà partie de l'environnement matériel des soignants. Le système enseignant, en s'engageant, un an auparavant dans cette médiation, a « repris en main » cette fiche, l'a modifiée et utilisée comme support d'apprentissages et de production d'écrits. Cette fiche recette, produite par les enfants et les enseignants spécialisés, a d'abord été ignorée par les infirmiers.
} 
L'élève, dans sa réponse, fait directement référence à l'apprentissage de la mesure (avec la notion de gramme) opérée lors du temps de la classe. La validation de l'infirmier n'est, quant à elle, possible que parce qu'il connaît les attentes des enseignants lors de la séance ; il sait que les pesées de $100 \mathrm{~g}$ font référence à la recette telle qu'elle a été travaillée dans le système d'enseignement. Les enseignants ont en effet proposé des mesures avec la balance électrique dont les pesées étaient inférieures ou égales à $100 \mathrm{~g}$. La notion de mesure est donc identifiée comme un objet de savoir, migrant d'un système à l'autre. Les professionnels utilisent une notion commune, reconnaissant ainsi qu'elle bénéficie aux savoirs et savoir-faire des élèves, dans les deux systèmes.

L'introduction de cette balance pour travailler la pesée fait donc naitre un environnement propice au changement. Ici, l'objet balance fait résonance entre les professionnels, il déstabilise juste ce qu'il faut les habitudes de travail, et modifie l'appréhension des objets ordinairement séparés des professionnels. L'association entre réunions d'ingénierie et événements de pratiques fait l'effet de déclencheur et engage alors les professionnels vers une modification de leur répertoire d'actions.

La fiche recette et l'objet balance n'ont pas été accueillis de la même manière dans les deux systèmes. Peut-être que la réappropriation de la fiche initialement issue du système de soin par le système auxiliaire classe a été perçue de manière intrusive par les infirmiers, provoquant un effet inverse à celui souhaité, c'est-à-dire un repli des infirmiers dans leur "zone sauvegardée et exclusive » (Emery, 2014). Ce que confirme cette affirmation d'un des infirmiers, au cours d'une réunion d'ingénierie : " Nous sommes dans un service de pédopsychiatrie ». Il marque ici l'importance qu'il porte à préserver une approche à dominante thérapeutique dans la prise en charge des enfants.

La balance est un nouvel objet dans chaque système, sa nouveauté rendait alors possible à chacun de s'en saisir, sans « froisser » l'autre. Cette nouveauté, au sens de territoire vierge, serait-elle alors une condition 1) pour construire un contexte cognitif commun et 2) d'enrichir les deux institutions ? L'objet, pour migrer, devrait donc faire signe à chacun, aux deux catégories de professionnels ET aux enfants. Le fait que la pesée soit un objet de savoir en cours d'acquisition en classe serait aussi gage de son passage d'un système à l'autre : il est appris dans un système (la classe) et utilisé dans l'autre (le soin). L'apprentissage en classe est alors évoqué dans l'espace de soin.

\section{L'ingénierie crée un contexte cognitif commun entre enseignants et infirmiers, déterminant pour accorder une plus grande part de responsabilité à l'élève.}

$\mathrm{Au}$ cours de la première réunion d'ingénierie, après le visionnage des vidéos, un infirmier interroge les autres professionnels : "Est-ce que, effectivement il ne faut pas qu'on fasse plus de lien entre nous pour avoir un langage commun pour que ce soit plus accessible pour eux [les enfants] aussi ?"

Si cet infirmier relève d'emblée qu'un langage commun aux adultes serait nécessaire, au fil des réunions d'ingénierie, cette idée va évoluer, se transformer et se concrétiser dans les pratiques, mais d'une manière qui va au-delà de l'idée première. Il dit en effet en fin de recherche : « je place l'enfant en position de savoir, en me mettant dans une position faussement naïve de lui demander comment on fait ». Il atteste désormais d'une plus grande réticence (il ne dit pas tout). Les énoncés de cet infirmier 
en T0 montraient une position initiale haute dans la topogénèse ${ }^{11}$, c'est-à-dire que l'infirmier prenait la responsabilité d'une grande part de l'activité ; Voici deux extraits, issus de T0.

"Je vais t'aider, je vais lire la première phrase : dans un saladier mélanger la fa... »(T0).

«Tu tiens le pot. Tu mets ton doigt là (sur le 150) et tu remplis jusque-là » (T0).

En T1 ses énoncés ont changé de nature.

"Tu veux lire toi-même? Alors vas-y» (T1).

«Alors maintenant il nous faut $100 \mathrm{~g}$ de beurre (...) Comment va-t-on faire?» (T1).

Cet infirmier adopte désormais une posture majoritairement d'accompagnement. L'existence, entre les deux systèmes, d'un contexte cognitif commun auquel soignants et enseignants spécialisés développent donc d'autres pratiques.

Sa manière de dévoluer la situation à l'élève pour qu'il en prenne la responsabilité est aussi tout autre. Il fait en effet référence au système d'enseignement et peut ainsi rappeler à la mémoire des enfants des éléments découverts et appris. Cette dévolution passe désormais par la formulation de questions alors qu'elles n'étaient pas du registre des interactions infirmier-enfant.

"Est-ce que tu te rappelles?"

"Est-ce que tu as besoin qu'on te rappelle?"

"Tu me racontes?"

"Tu as vu ça à l'école je crois?»

"Tu veux bien m'expliquer comment va-t-on faire?"

"Comment as-tu appris?»

Ces questions traduisent explicitement les liens entre les systèmes : les savoirs enseignés dans le système d'enseignement font signe pour les infirmiers.

Avec cette nouvelle forme de langage, l'infirmier opère ainsi un transfert de responsabilités aux élèves, de leurs savoirs et de leurs actions. Ces questions disent symboliquement à l'élève que la balle est dans son camp et la transformation -ici la réalisation de la recette- de son fait.

Cette manière de faire contribue aux attentes initiales qui voulaient, dans le système d'enseignement, placer les enfants dans une posture d'apprentissage et les consolider, et, dans le système de soin, une reprise de confiance en soi.

L'asymétrie initiale entre les deux systèmes se joue désormais différemment au fur et à mesure qu'une alliance entre professionnels s'opère ; on assiste alors à un rééquilibrage entre les deux systèmes. Le système auxiliaire d'enseignement, n'est plus considéré comme intrusif pour le système de soin et les apprentissages qui sont effectués avec les enseignants sont explicitement appelés à être recontextualisés dans le système soin. Ce qui était perçu, à l'origine, comme un empiètement du système enseignement dans le système soin n'existe plus.

\section{Conclusion}

L'évolution des pratiques vers une coopération des professionnels et la construction d'une culture commune s'est effectuée au cours de cette ingénierie coopérative entre acteurs de terrain et chercheur.

${ }^{11}$ Place de l'acteur par rapport aux savoirs. 
Le décret $n^{\circ}$ 2009-378 envisage la coopération comme une «logique de projet qui exige des individus et des structures qui les emploient de donner par eux-mêmes sens à l'entreprise et de réunir les conditions permettant que les interactions puissent avoir un caractère organisationnel suffisamment fort pour que les activités et les tâches soient inscrites dans un système » (Ebersold, 2012). Certes ce caractère organisationnel est obligatoire, mais nous l'avons vu, il ne va pas de soi. Des conflits ayant comme origine les «savoirs professionnels » (Perrenoud, 2001) mis en place au cours de l'activité restent en effet, de prime abord, fortement marqués par les empreintes professionnelles initiales. De ce fait, notre recherche montre qu'une coopération n'est pas envisageable tant que la notion de territoires respectifs n'est pas remise en question. "Le système de soin doit pouvoir interagir avec le système éducatif. Aussi un temps de formation commun est-il nécessaire pour s'inscrire résolument dans une perspective inclusive " (De Anna et al., 2008). Une "zone interprétative partagée » (Desgagné et al., 2001) représentée par l'ingénierie coopérative, est devenue un lieu de construction de savoirs communs sur la pratique des différents professionnels et a écarté la crainte d'une perte d'identité. Elle a aussi fait prendre conscience aux professionnels des avantages des pratiques coopératives dans les prises en charge des enfants à besoins particuliers. Le travail dans le système enseignement-apprentissage a en effet produit de nouveaux savoirs qui sont devenus des ressources dans le système de soin (et inversement ${ }^{12}$ ). Au-delà du partage de territoire, il existe en effet un partage des tâches qui «confère une unité d'actions sans annuler la diversité et autorise ainsi des modalités de coopération où les intérêts interindividuels et collectifs s'interpénètrent » (Ebersold, 2012). Cette «médiation cuisine », considérée comme tiers dans le rapport enseignants/infirmiers, via d'objets de savoirs migrants facilite alors une articulation des pratiques professionnelles et les savoirs, savoir-faire et savoir-être des enfants.

\section{Références}

Altet, M. (2000). L'analyse de pratiques: une démarche de formation professionnalisante ? Recherche \& formation, 35(1), 25-41.

Benoit, H. (2012). Pluralité des acteurs et pratiques inclusives : les paradoxes de la collaboration. $L a$ nouvelle revue de l'adaptation et de la scolarisation, 57(1), 65-78.

Brousseau, G. (1998). Théorie des situations didactiques, en mathématiques. Grenoble: $\quad$ La pensée sauvage.

Chauvière, M., \& Plaisance, É. (2008). Les conditions d'une culture partagée. Reliance, (1), 31-44.

Chevallard,Y. (1995). La fonction professorale : esquisse d'un modèle didactique. In Noirfalise et Perrin-Glorian (dir.), Actes de la VIIIe école d'été de didactique des mathématiques_(pp. 83-122). Clermont-Ferrand : IREM.

Dorison, C. (2015). La question de la scolarisation dans les établissements spécialisés en France : 1975-2009. La nouvelle revue de l'adaptation et de la scolarisation, (2), 67-78.

Desgagné, S., Bednarz, N., Lebuis, P., Poirier, L., \& Couture, C. (2001). L'approche collaborative de recherche en éducation : un rapport nouveau à établir entre recherche et formation. Revue des sciences de l'éducation, 2001/1 (27), 33-64.

\footnotetext{
${ }^{12}$ Mais nous n'avons pas centré ce présent article sur ce que le système soin produit sur le système enseignementapprentissage.
} 
Duquesne-Belfais, F. (2008). Répondre aux exigences de la formation des enseignants de l'ASH. L'analyse de pratiques comme lien entre théorie et pratique. La nouvelle revue de l'adaptation et de la scolarisation, 41(1), 11-24.

De Anna, L., Walter, B. \& Jeanne, Y. (2008). Les processus d'intégration scolaire en Italie et la formation des enseignants. Reliance, 27, (1), 111-120.

Ebersold, S. (2012). Parcours de scolarisation et coopération : enjeux conceptuels et méthodologiques. La nouvelle revue de l'adaptation et de la scolarisation, 2012/1 (57), 55-64.

Emery, R. A. (2011). L'équipe interprofessionnelle comme outil privilégié de l'accompagnement des enfants et adolescent-e-s présentant des besoins particuliers. Revue Suisse De Pédagogie Spécialisée. https://www.csps.ch/bausteine.net/file/showfile.aspx?downdaid=8551\&guid=01268ff2-76e7-49e1$a 39 b-f 4 c 721130 a 7 a \& f d=0$

Emery, R. (2014). Un langage commun, condition du travail en équipe multiprofessionnelle ? $L a$ nouvelle revue de l'adaptation et de la scolarisation, 65 (1), 41-53.

Emery, R. (2017). La collaboration multiprofessionnelle dans les contextes d'institution spécialisée. La nouvelle revue de l'adaptation et de la scolarisation, (2), 193-209.

Emery, R. A., \& Pelgrims, G. (2015). Collaboration multiprofessionnelle dans un contexte d'enseignement spécialisé : activité déployée pour la conception, la mise en œuvre et la régulation d'un projet d'élève. Recherches en éducation, (7), 43-53.

Hochmann, J. (2013). Pour soigner l'enfant autiste. Paris : Odile Jacob.

Lieberman, A. (1986). Collaborative research: Working with, not working on. Educational leadership, 43(5), 28-32.

Marciano, P. (2009). L'hôpital de jour pour enfants : Dans le parcours de soin. Eres.

Marlot, C., Toullec-Théry, M., \& Daguzon, M. (2017). Processus de co-construction et rôle de l'objet biface en recherche collaborative. Phronesis, 6(1), 21-34.

Perrenoud, P. (2001). Mettre la pratique réflexive au centre du projet de formation. Cahiers enseignement-apprentissages, (390), 42-45.

Perraud, C. (2018). Une ingénierie coopérative : des travailleurs, des professionnels et un chercheur dans le secteur du travail protégé (Esat). Une enquête collective pour une amélioration des pratiques. Thèse de doctorat. Université de Bretagne occidentale, Brest, France.

Ruchat, M. (2003). Histoire de l'éducation spéciale : une spécificité plurielle. 155-169. De Boeck Supérieur.

Sensevy, G. (2007). Des catégories pour décrire comprendre l'action didactique -in G.SENSEVY, G, A. MERCIER. Agir ensemble. L'action conjointe du professeur et des élèves, Rennes, PUR, p. 13-49. Sensevy, G. (2011). Le sens du savoir. Éléments pour une théorie de l'action conjointe en didactique. Bruxelles : De Boeck.

Sensevy, G., \& Quilio, S. (2002). Les discours du professeur. Vers une pragmatique didactique. Revue française de pédagogie, 47-56.

Tambone, J. (2014). Enseigner dans un dispositif auxiliaire : le cas du regroupement d'adaptation et de sa relation avec la classe d'origine de l'élève. Les Sciences de l'éducation - Pour l'Ére nouvelle, 2014/2 (47), 51-71. 